\title{
Outcome of probing and syringing in congenital nasolacrimal duct obstruction at various age groups in a tertiary eye center in Western Nepal
}

\author{
Koshal Shrestha1, Binita Bhattaraiं ${ }^{2}$ Laxmi Devi Manandhar ${ }^{3}$, Salma KC Rai ${ }^{4}$, \\ Araniko Pandey ${ }^{5}$, Shanti Gurung ${ }^{6}$ \\ ${ }^{1,2}$ Consultant Oculoplastic Surgeon, ${ }^{4}$ Professor and Consultant Pediatric Ophthalmologist, ${ }^{3,5,6}$ Ophthalmologist, \\ Lumbini Eye Institute
}

Background: Congenital nasolacrimal duct obstruction (CNLDO) is one of the commonest problems encountered in daily ophthalmology practice. Though probing and syringing is the mainstay of management of CNLDO, there is no clear guideline about the optimal time to perform. Aims and Objective: To evaluate the efficacy of probing and syringing in cases of congenital nasolacrimal duct obstruction in various age groups. Materials and Methods: In this prospective, hospital based, interventional, cross-sectional study done over a period of 2 years (July 2015- June 2017), children of various age ranging from 10 days to 8 years who underwent probing and syringing under general anesthesia for CNLDO refractory to conservative measures were included. Results: Children of as young as 10 days to as older as 8 years underwent probing and syringing with male to female ratio of 1.2:1. Overall success was $80.6 \%(58 / 72)$ which was statistically significant when analyzed with age $(P=0.006)$. Majority of cases with successful outcome were below 24 months of age (90.62\%). There was predominance of membranous obstruction (128/194) over bony obstruction (66/194). Conclusions: Nasolacrimal duct probing under GA is a safe and viable option as a primary treatment modality for CNLDO. Though the success of probing is high in the younger children, it's worthwhile to consider the same in older children at least up to 5 years of age before going for more invasive procedure like DCR surgery since more than 2 third of probing has successful outcome up to that age.

Key words: Congenital nasolacrimal duct obstruction; Probing and syringing; Age group
http://nepjol.info/index.php/AJMS DOI: 10.3126/ajms.v9i6.20508 E-ISSN: 2091-0576 P-ISSN: 2467-9100

\section{INTRODUCTION}

Congenital nasolacrimal duct obstruction (CNLDO) is one of the commonest problems encountered in daily ophthalmology practice. It can be unilateral or bilateral with no reported sex or genetic predilection. CNLDO is prevalent in approximately $6 \%$ of new born with wide variation of $1.2 \%$ to $30 \%$ due to failure of canalization of nasolacrimal duct mostly at the lower end. ${ }^{1,2}$ However, in as many as $90 \%$, the membrane that obstructs valve of hasner dissolves spontaneously in the first 6 months with or without conservative treatment comprising of sac massage, lid hygiene and topical antibiotics. ${ }^{3}$

The causes of failure of long term conservative treatment include poor parent's compliance, faulty technique of hydrostatic massage, poor hygiene. However, obstruction type may also play the role in the outcome as well. Along with bothersome watering as the prime issue, CNLDO may cause repeated attacks of conjunctivitis, dacryocystitis, mucocele or pyocele and lacrimal fistula formation as well. Beside these ocular problems watering due to CNLDO may 
affect both the children and the parents psychologically and socially.

Children usually present with watery and sticky eye, matted eyelashes, discharge, skin. Regurgitation on pressure over lacrimal sac and/or positive fluorescence usually confirm the diagnosis. ${ }^{4}$ However, it is crucial to rule out the other causes of epiphora such as lid and lash abnormalities and corneal abrasions, congenital glaucoma.

Probing under topical or general anesthesia (GA) is standard management of CNLDO who does not respond the conservative treatment. ${ }^{5}$ Studies have found probing to be successful in $70 \%$ to $97 \%$ of CNLDO. ${ }^{1,6,7}$

The question about the optimal time for probing remains unsolved. Some authors believes that delay in probing beyond 1 year is associated with a lower rate of success and this worsens with increasing age ${ }^{1,6,8}$ which could be, perhaps, due to prolonged inflammation and fibrosis in the lacrimal duct system. Moreover the apparent decline in success rate in older children is due to accumulation of more complex obstruction as less severe obstruction clears spontaneously., Earlier intervention may prevent the occurrence of prolonged inflammation in the lacrimal drainage system and avoid its possible complications and inconvenience. However, others believe that primary probing continues to be an effective treatment well beyond 2 years of age and that the cure rate does not vary markedly with age. ${ }^{10,11}$ There are, thus no clear guidelines for management of congenital nasolacrimal duct obstruction, in terms of probing, especially for older children.

There are very few studies from other parts of Nepal. So, with this study we aimto evaluate the cure rate and assess the factors affecting the cure rate of probing and syringing for CNLDO among the children of wide age range in a tertiary eye hospital in western Nepal.

\section{MATERIALS AND METHODS}

This study was conducted between July 2015 and June 2017, A total of 194 eyes of 149 consecutive patients, 81 males and 68 females, aged between 10 days to 96 months old who underwent probing of the nasolacrimal system for CNLDO, not responsive to conservative measures, were included into the study. The cases of epiphora with history of trauma to the nasolacrimal system, punctal or canalicular abnormalities, craniofacial anomalies, and abnormalities of the eyelids were excluded from the study. Ethical approval was obtained from the Institutional Research Review Board of Lumbini Eye Institute. A written informed consent was obtained.
The diagnosis of $\mathrm{CNLDO}$ was clinical, as evidenced by history of epiphora beginning during the first few weeks of life, noninflamed conjunctiva, crusting, increased tear meniscus height, recurrent mucopurulent discharge, and regurgitation on pressure over lacrimal sac (ROPLAS) along with fluorescein dye disappearance test (FDDT) whenever necessary.

Probing was performed after 2 to four-week course of conservative treatment including proper hydrostatic Crigler lacrimal massage and topical antibiotics. Acute dacryocystitis were treated conservatively and cases of lacrimal abscess were treated first by incision and drainage several days before taking for probing.

The probing was performed under GA with a laryngeal mask airway. Throat pack with a moistened soft cotton gauge roll was done in each case to absorb excess fluid from lacrimal irrigation. Probing was carried out through the lower punctum using Bowman probe of appropriate size for individual patient starting from $0000(0.70-\mathrm{mm}$ diameter) to $0(1.00-\mathrm{mm}$ diameter $)$ size decided on the table.

Once the nasolacrimal canal was entered, the probe was gently advanced until resistance was encountered. Firm pressure was then applied to the probe, attempting to advance it beyond the obstruction. The types (membranous or firm) of the obstruction of nasolacrimal duct were noted in each case. A membranous obstruction was characterized by lack of or minimum resistance to the probe at the lower portion of the nasolacrimal duct that is overcame without much effort where as it is classified as hard or firm type if it needs much effort to overcome the obstruction.

The patency of the nasolacrimal system was evaluated by irrigation of fluorescein stained saline through the lower punctum and confirmed by the direct visualization of stained fluid in the ipsilateral nostril or by seeing yellowish staining of throat pack.

Each post probing child received steroid-antibiotic eye drop four times a day for 2 weeks along with lacrimal sac massage till next follow up or until the symptoms disappears.

Duration of follow-up for all patients was at least 2 weeks after the probing. Each patient was evaluated at around 2 weeks, 6 weeks, and 10 weeks after probing whenever possible.

Success of probing was the main outcome measure and was predefined as complete remission of watering, discharge or reflux of sac content on pressure within 2-6 weeks of the procedure. At least two attempts of probing at least 6 weeks apart, if the first attempt fails, was mandatory 
before the procedure was declared as failure. All the data was collected on a proforma and the results were analyzed using SPSS version 20.0. Analysis was performed using the chi-square test. Statistical significance was set at $\mathrm{p}<0.05$.

\section{RESULTS}

During 2 years of study period (July 2015 - June 2017), a total of 194 eyes of 149 children with CNLDO, including 81 boys and 68 girls (Table 1) were divided into six age groups e.g.0-1 year, $1-2$ years, 2-3 years, 3-4 years, 4-5 years and more than 5 years (Table 2). Mean age of the children at the time of intervention was 31.83 months (SD \pm 21.52 ) years ranging from 10 days to 8 years (Table 1 ).

About $71.8 \%$ of children ( $n=107 / 149)$ had onset of the symptoms within 2 weeks of age where as $28.2 \%$ (42 children) developed symptoms 2 weeks or more after birth. Unilateral obstruction was present in 104 children (69.8\%, Right Eye 60 and Left Eye 44), while bilateral obstruction was present in 45 other children $(30.2 \%)$. The most common signs were epiphora with or without discharge, crusting on the lids, mattering of lashes and macerated eczematous lid skin, sac swelling or lacrimal abscess. There was regurgitation of mucoid/mucopurulent material with pressure over the lacrimal sac in the majority of subjects.

The success of the initial probing was $78.9 \%(153 / 194)$ which escalated up to $82.5 \%(160 / 194)$ after repeat probing (Table 2). Five children out of 32 of failed initial probing got cured after repeat probing and one child improved. Successful initial or repeat probing noticed more in younger age group (Table 2) and declined with the increasing age which was clinically significant $(\mathrm{p}=0.002$ and 0.001$)$.
There was higher requirement of repeat probing among the bony obstruction (36\%) as compared to membranous obstruction $(11.7 \%)$ which was clinically significant $(\mathrm{p} \leq 0.001)$ (Table 3).

The mean distance between punctum to nasal floor measured as the length of probe was $30.78 \mathrm{~mm}$ (SD $3.63 \mathrm{~mm}$ ) with minimum $23 \mathrm{~mm}$ and maximum $40 \mathrm{~mm}$.

\section{DISCUSSION}

Congenital nasolacrimal duct obstruction (CNLDO) manifests at birth due to the failure of canalization of the ectodermal cord of cells. Regular and frequent massage is the primary treatment by which $95 \%$ of the cases resolve during the first year of life. Probing is the method of choice for the treatment of CNLDO, non-responsive to conservative treatment. However, the timing of probing has always been a topic of debate. Early probing has been advocated at presentation or shortly after a period of conservative treatment irrespective of the age of the child. Probing is usually performed at or after 1year age, but as the child grows, resistance to the passage of the probe results in higher failure rates in older children.

Ffookes recommends early probing of the nasolacrimal system, after a short course of topical antibiotic therapy to avoid the complications like acute or recurrent dacryocystitis or canaliculitis or the formation of lacrimal abscess and to free the child and parents of the inconvenience of persistent epiphora, discharge and recurrent infections. ${ }^{1,12-14}$ Moreover, some authors believes that delayed probing beyond 1 year may be associated with lower cure rates because of fibrosis due

\section{Table 1: Patients demography and laterality of involvement}

\begin{tabular}{|c|c|c|c|c|c|}
\hline \multirow[t]{2}{*}{ Sex of the patients } & \multicolumn{3}{|c|}{ Laterality } & \multirow[t]{2}{*}{ Total patients } & \multirow[t]{2}{*}{ Total eyes } \\
\hline & Right & Left & Bilateral & & \\
\hline Male $(81,54.36 \%)$ & $31(38.3 \%)$ & $23(28.4 \%)$ & $27(33.3 \%)$ & $81(54.36 \%)$ & $108(55.67 \%)$ \\
\hline Female $(68,45.63 \%)$ & 29 (42.6\%) & $21(30.9 \%)$ & $18(26.5 \%)$ & 68 (45.63\%) & $86(44.32 \%)$ \\
\hline Total & $60(40.3 \%)$ & $44(29.5 \%)$ & $45(30.2 \%)$ & $149(100.0 \%)$ & $194(100 \%)$ \\
\hline
\end{tabular}

\section{Table 2: Success of probing according to the age group}

\begin{tabular}{|c|c|c|c|c|}
\hline \multirow[t]{2}{*}{ Age category (month/s) } & \multicolumn{2}{|c|}{ Success after single probing } & \multicolumn{2}{|c|}{ Overall success } \\
\hline & yes & no & Yes & No \\
\hline 0 to $12(n=53)$ & $47(88.7 \%)$ & $611.3 \%)$ & $49(92.5 \%)$ & $4(7.5 \%)$ \\
\hline 13 to $24(n=57)$ & $51(89.5 \%)$ & $6(10.5 \%)$ & 52 (91.2\%) & $5(8.8 \%)$ \\
\hline 25 to $36(n=34)$ & $25(73.5 \%)$ & $9(26.5 \%)$ & $27(79.4 \%)$ & $7(20.6 \%)$ \\
\hline 37 to $48(n=15)$ & $9(60.0 \%)$ & $6(40.0 \%)$ & $11(73.3 \%)$ & $4(26.7 \%)$ \\
\hline 49 to $60(n=17)$ & $11(64.7 \%)$ & $6(35.3 \%)$ & $11(64.7 \%)$ & $6(35.3 \%)$ \\
\hline$>60(n=18)$ & $10(55.6 \%)$ & $8(44.4 \%)$ & $10(55.6 \%)$ & $8(44.4 \%)$ \\
\hline Total 9N=194) & $153(78.9 \%)$ & $41(21.1 \%)$ & $160(82.5 \%)$ & $34(17.5 \%)$ \\
\hline$P$ value & \multicolumn{2}{|c|}{$p$-value $=0.002$} & \multicolumn{2}{|c|}{$p$-value $=0.001$} \\
\hline
\end{tabular}




\begin{tabular}{|c|c|c|c|c|}
\hline \multirow[t]{2}{*}{ Type of obstruction on probing } & \multicolumn{2}{|c|}{ Success after first time probing } & \multicolumn{2}{|c|}{ Overall success } \\
\hline & Yes & No & Yes & No \\
\hline Membranous $(n=128)$ & $113(88.3 \%)$ & $15(11.7 \%)$ & $116(90.6 \%)$ & $12(9.4 \%)$ \\
\hline Bony $(n=66)$ & $40(60.6 \%)$ & $26(39.4 \%)$ & $44(66.7 \%)$ & $22(33.3 \%)$ \\
\hline Total $(n=193)$ & $153(78.9 \%)$ & $41(21.1 \%)$ & $160(82.5 \%)$ & $34(17.5 \%)$ \\
\hline$p$-value & \multicolumn{2}{|c|}{$<0.001$} & \multicolumn{2}{|c|}{$<0.001$} \\
\hline
\end{tabular}

to prolonged inflammation in the lacrimal drainage system with increasing age. ${ }^{7,9}$

In our study, the initial success rate was $78.9 \%$ which escalated to overall success of $82.5 \%$ with repeat probing which is comparable to previous studies. ${ }^{1,15-17} \mathrm{We}$ found a significant trend of decreasing success rates (overall) with increasing age: $92.5 \%, 91.2 \%, 79.4 \%, 73.3 \%, 64.7 \%$ and $55.6 \%$ at $0-1$ year, 1-2 years, 2 to 3 years, 3 to 4 years, $4-5$ years and more than 5 years respectively which is consistent with other studies. ${ }^{8,15,16}$ Kashkouli et $\mathrm{al}^{7}$ concluded that older children are more likely to have complicated, non-membranous obstructions that might reduce the cure rate. In contrast, El-Mansoury and associates ${ }^{11}$ in their study of 138 children ranging from 13 months to 7 years, found more than $90 \%$ cure rate regardless of age. Robb reported similar data, reflecting a uniform cure rate of nearly $90 \%$ with the first-time probing in children ranging in age from 1 to 9 years old and noticed that altered anatomy is important in determination of failure. ${ }^{18}$ In current study, we noticed favorable outcome in cases of membranous obstruction at the lower end of nasolacrimal duct consistent to the study of Kushner. ${ }^{19}$

Most of our study population (71.1\%) had onset of symptoms like tearing, discharge, matted lashes, swelling over the lacrimal sac region within 2 weeks of birth and $28.9 \%$ had symptoms developed after 2 weeks of age as noticed by Ballard. ${ }^{16,20}$ However Ffookes ${ }^{12}$ reported that 188 out of 443 cases developed symptoms during one week after birth which may be due to reflex tear production from inflammation of the lacrimal sac.

Our result shows higher success rate in cases of membranous obstruction indicating the anatomic basis of outcome of probing. The majority of obstructions were felt at the lower end of the NLD. However, we could not comment on the exact site of obstruction and the condition at the site of the valve of Hasner, as we didn't use nasal endoscope. Though endoscope may provide better visualization of the nature of distal blockage (i.e., stenosis, atresia, inferior turbinate position) and direct observation of the probe and fluorescein outflow ${ }^{21,22}$ it does not alter the outcome significantly. ${ }^{23}$

In the present study, a Bowman probe of largest appropriate size ranging from size 000 to size 1 was used.
Many authors recommend using a specific size of Bowman probe despite of the fact that there is no standardization among instrument manufacturers with respect to the size of the Bowman probe. ${ }^{16}$

Previously probing in children was usually performed under topical anesthesia as office procedure which may be due to lower availability of anesthesia service in their center. ${ }^{6,24,25}$ In our study, all probing procedures were performed under general anesthesia as recommended by many authors because it provides better control over the procedure and paying attention to the site and nature of the obstruction. ${ }^{26}$ It also reduces the potential risk of trauma to delicate structures of the lacrimal drainage system and soothed the apprehension of the child and parents. We could measure and found the average punctum to nasal floor distance $(30 \mathrm{~mm})$ by measuring the fraction of the probe passed through the punctum to reach the nasal floor which was longer than described in a only one study which may probably be due to large age range in our s study. ${ }^{27}$ However, it would be nicer to measure the distance between the punctum to the obstruction point.

The successful outcome of single or repeat probing at 2 weeks post-operative follow up was highly correlated with the final results at 6 months or more as observed by Perveen $\mathrm{S}$, et $\mathrm{al}^{16}$ indicating that the early result could represent the final result of probing.

\section{CONCLUSION}

Nasolacrimal duct probing under GA is a safe and viable option as a primary treatment modality for CNLDO. Though the success of probing is high in the younger children, but in cases of delayed presentation, it would be worth to consider the same in older children at least up to 5 years of age before undertaking for DCR surgery since more than 2 third children has successful probing. Simple membranous obstructions havefavorable probing outcome than in cases of bony obstruction.

\section{ACKNOWLEDGEMENTS}

The authors would like to thank Mr. Tanka Gaire for filling the proforma and making files and Mr. Ganesh 
Thapa for keeping medical records of study population and Mrs. Geeta Shrestha for her assistance in drafting the manuscript. The authors also would like to thank Ms. Mina KC and Ms. Sujata Shrestha for their support during procedure in operating room.

\section{REFERENCES}

1. Katowitz JA and Welsh MG. Timing of initial probing and irrigation in congenital nasolacrimal duct obstruction. Ophthalmology 1987;94(6):698-705.

2. Wagner RS. Lacrimal disorders. Ophthalmol Clin North Am 1996; 9: 229-237.

3. Nelson LR, Calhoun JH and Menduke H. Medical management of congenital nasolacrimal duct obstruction. Ophthalmology 1985;92(9):1187-1190.

4. Ahmed I, Saleem Z and Ahmed W. Outcome of nasolacrimal probing without syringing in congenital nasolacrimal duct obstruction under inhalational anaesthesia in infants of 6-12 months of age.PJMHS 2013;17(4): 1113-1117.

5. Honavar SG, Prakash VE and Rao GN. Outcome of probing for congenital nasolacrimal duct obstruction in older children. Am J Ophthalmol 2000;130(1):42-48.

6. Stager D, Baker JD, Frey T, Weakley DR and Birch EE. Office probing of congenital nasolacrimal duct obstruction. Ophthalmic Surg 1992;23(7):482-484.

7. Kashkouli MB, Beigi B, Parvaresh MM, Kassaee A and Tabatabaee $Z$. Late and very late initial probing for congenital nasolacrimal duct obstruction: what is the cause of failure? $\mathrm{Br} \mathrm{J}$ Ophthalmol 2003;87(9):1151-1153.

8. Mannor GE, Rose GE, Frimpong-Ansah $K$ and Ezra E. Factors affecting the success of nasolacrimal duct probing for congenital nasolacrimal duct obstruction. Am J Ophthalmol 1999;127(5):616-617.

9. Robb RM. Success rates of nasolacrimal duct probing at time intervals after 1 year of age1. Ophthalmology 1998;105(7):1307-1310.

10. Kushner BJ. The management of nasolacrimal duct obstruction in children between 18 months and 4 years old. J AAPOS Off Publ Am Assoc PediatrOphthalmol Strabismus 1998;2(1):57-60.

11. El-Mansoury J, Calhoun JH, Nelson LB and Harley RD. Results of late probing for congenital nasolacrimal duct obstruction. Ophthalmology 1986;93(8):1052-1054.
12. Ffooks $\mathrm{OO}$. Dacryocystitis in Infancy. $\mathrm{Br} \mathrm{J}$ Ophthalmol 1962;46(7):422-434.

13. Ffooks $\mathrm{OO}$. Lacrimal abscess in the newborn. $\mathrm{Br} \mathrm{J}$ Ophthalmol 1961;45(8):562-565.

14. MacEwen C, Young J, Barras C, Ram B and White P. Value of nasal endoscopy and probing in the diagnosis and management of children with congenital epiphora. $\mathrm{Br} J$ Ophthalmol 2001;85(3):314-318.

15. Kashkouli MB, Kassaee A and Tabatabaee Z. Initial nasolacrimal duct probing in children under age 5: cure rate and factors affecting success. J AAPOS Off Publ Am Assoc PediatrOphthalmol Strabismus 2002;6(6):360-363.

16. Perveen S, Sufi AR, Rashid S and Khan A. Success Rate of Probing for Congenital Nasolacrimal Duct Obstruction at Various Ages. J Ophthalmic Vis Res 2014;9(1):60-69.

17. Kim YS, Moon SC and Yoo KW. Congenital nasolacrimal duct obstruction: irrigation or probing? Korean J Ophthalmol 2000;14(2):90-96

18. Robb RM. Probing and Irrigation for Congenital Nasolacrimal Duct Obstruction. Arch Ophthalmol1986;104(3):378-379.

19. Kushner BJ. The management of nasolacrimal duct obstruction in children between 18 months and 4 years old. J AAPOS Off Publ Am Assoc PediatrOphthalmol Strabismus 1998;2(1):57-60.

20. Ballard EA. Excessive tearing in infancy and early childhood. The role and treatment of congenital nasolacrimal duct obstruction. Postgrad Med 2000;107(6):149-154.

21. Paul TO. Medical management of congenital nasolacrimal duct obstruction. J PediatrOphthalmol Strabismus 1985;22(2):68-70.

22. Petersen RA and Robb RM. The natural course of congenital obstruction of the nasolacrimal duct. J Pediatr Ophthalmol Strabismus 1978;15(4):246-250.

23. Zilelioğlu $\mathrm{G}$ and Hoşal $\mathrm{BM}$. The results of late probing in congenital nasolacrimal duct obstruction. Orbit AmstNeth 2007;26(1):1-3.

24. Cha DS, Lee H, Park MS, Lee JM and Baek SH. Clinical outcomes of initial and repeated nasolacrimal duct office-based probing for congenital nasolacrimal duct obstruction. Korean J Ophthalmol 2010;24(5):261-266.

25. Shrestha JB, Bajimaya $S$ and Hennig A. Outcome of probing under topical anesthesia in children below 18 months of age with congenital nasolacrimal duct obstruction. Nepal Med Coll Journal 2009;11(1):46-49.

26. Macewen CJ. Congenital nasolacrimal duct obstruction. ComprOphthalmol Update 2006;7(2):79-87.

27. Bageja S, Grover AK and Bansal M. Congenital nasolacrimal duct obstruction. DOS Times 2007;12(10):1-5.

\footnotetext{
Authors Contribution:

KS-Concept and design of the study, literature review, Data collection and analysis and revision of manuscript; BB-Literature review, Editing the manuscript; LDM-Data analysis, Editing the manuscript; SKC-Critical revision of the manuscript; AP-Editing the manuscript; SG- Editing the manuscript.

Work attributed to:

Department of Orbit and Oculoplasty Lumbini Eye Institute Bhairahawa, Nepal.

Orcid ID:

Dr.Koshal Shrestha- (D) http://orcid.org/0000-0002-5640-6590

Dr.Binita Bhattarai- 1 http://orcid.org/0000-0002-0397-7699

Dr. Laxmi Devi Manandhar- 1 http://orcid.org/0000-0002-6029-9337

Dr. Salma KC Rai- (1) http://orcid.org/0000-0001-5559-1512

Dr. Araniko Pandey- (D) htp:///orcid.org/0000-0002-0791-293X

Dr. Shanti Gurung- (1) http://orcid.org/0000-0001-8408-4245

Source of Support: Nil, Conflict of Interest: None declared.
} 